\title{
MPLS L2-VPN using Atom with Like to Like Circuit using Ethernet at Layer 2
}

\author{
Aarthi.M ${ }^{1}$, Suganya. ${ }^{2}$ \\ Assistant Professor, Department of Computer Science, \\ Ponnaiyah Ramajayam Institute of Science and Technology PRIST University Thanjavur ${ }^{1}$ \\ M.C.A., Scholar Department of Computer Science, \\ Ponnaiyah Ramajayam Institute of Science and Technology PRIST University, Thanjavur ${ }^{2}$
}

\begin{abstract}
MPLS is the prime technology used in examine supplier Networks as quick pack forwarding mechanism. It is the tools used in service supplier networks to connect dissimilar remote sites. MPLS can be used to carry any kind of information whether it is layer 2 data such as frame relay, Ethernet, ATM data or layer 3 data such as IPV4, IPV6. MPLS creates two types of VPNs. One is Layer 3 MPLS VPN with Layer 2 MPLS VPN. In Layer 3 MPLS VPN, customer forms IP national distribute with Service Provider device. In Layer 3 VPN routing is performed between client edge device and Provider Edge device. Layer 2 VPNs behave like the consumer sites are connected using a Layer 2 Switch. Various L2 MPLS VPN techniques are Virtual Private LAN Service (VPLS), Virtual Private Wire Service (VPWS), and Ethernet VPN. This paper gives an indication of all these L2 and L3 MPLS VPN technologies.
\end{abstract}

Keywords: MPLS, VPLS, VPWS, Atom

\section{INTRODUCTION}

MPLS VPN is the internet/intranet connection of the client to client who are geographically separated. MPLS VPN circuits are interconnected from one Service providers to another Service providers by using Exterior Border Gateway Protocol (EBGP). Data is forwarded between client to customer using the MPLS-enabled Service Provider IP backbone .Customer need not worry about the geographical locations. They can get MPLS VPN service any time anywhere. Previously all the network routers of ISP are operating with Routing Protocols which will create routing tables based on IP Pool networks. As the networks were growing exponentially with huge evolution of internet, the routers routing process is getting delayed and latency also increased.

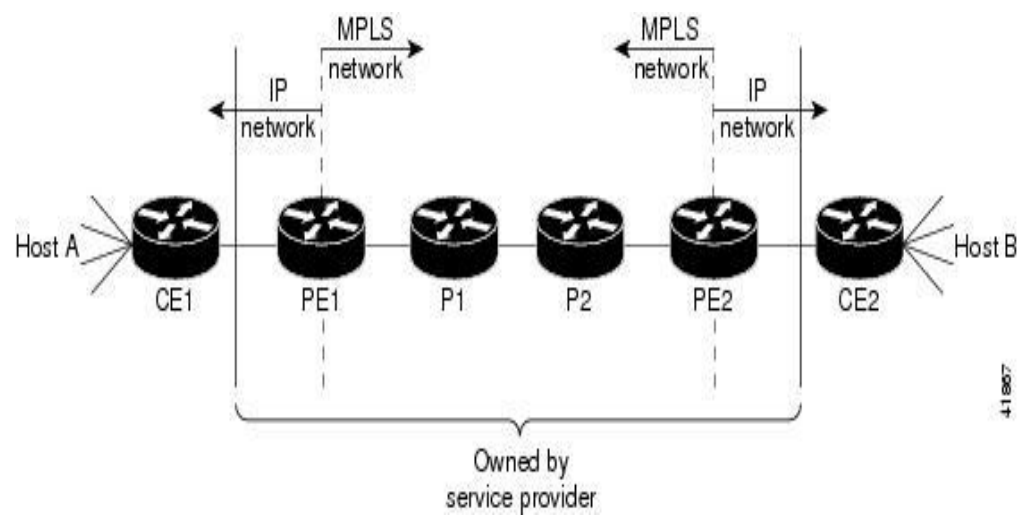

\section{Components of MPLS IP Network:}

- $\quad$ Client Edge, which workings at IP level.

- $\quad$ Supplier Edge is the entry point of MPLS Domain.

- $\quad$ Supplier Routers are works as transit switches in between LERs.

- Label switched path is the data trail between two routers, through which packets are traveling. 


\section{LITERATURE REVIEW}

MLS Architecture [1] by E. Rosen of Cisco system, A. Viswanathan of Force10 network, and R. Callon of Juniper Networks in Internet Engineering Task Force (IETF) RFC - 3031 specifies the structural design of MPLS. It is the first typical document of MLS by IETF MPLS operational Group. Framework for Layer 2 Virtual Private Networks (L2VPNs) [2] by L. Andersson, Ed. , Acreo AB, E. Rosen, Ed. Of Cisco Systems provides a framework for Layer 2 provider provisioned Virtual Private Networks (L2VPNs). This framework is planned to aid in standardize protocols and mechanism to maintain interoperable L2VPNs. This model also is a ordinary document for Virtual Private Wire Service (VPWS) and Virtual Private LAN Service(VPLS). Pseudo line Setup and upholding Using the Label Distribution Protocol (LDP) [3] by L. Martini, E. Rosen of Cisco Systems, N. Eul-Aawar of Level 3 Communications, T. Smith of system Appliance and G. Heron of Tellabs describe how layer 2 services like Frame Relay, Asynchronous transport method, and Ethernet can be emulate over a MPLS backbone by encapsulate the Layer 2 protocol units (PDU) and transmit them over "pseudo wires". This document specifies a protocol for establishing and maintaining the pseudo wires, using extensions to LDP. Encapsulation Methods for express of Ethernet over MPLS network [4] by L. Martini, Ed. , E. Rosen of Cisco Systems, N. El-Aawar of Level 3 Communications and G. Heron of Tellabs describes an Ethernet pseudo wire(PW) is used to carry Ethernet/802.3 protocol data units(PDUs) over an MPLS network. VPLS Using BGP for Auto-Discovery and signal [5] by K. Komepella, Ed. And Y. Rekhter, Ed of Juniper Networks describes BGP Auto Discovery and Signaling method for VPLS. It specifies a device for signal a VPLS, and convention for forward VPLS frame across a packet switched network.

\section{EXISTING SYSTEM}

\section{MPLS Layer 2 VPN}

Virtual Private Wire Service (VPWS) / Any transport over MPLS (AToM):

AToM is Cisco's execution of VPWS in MPLS network that provides Point-to-Point tunnel service from PE to PE. Two types of pseudo wire technology are used in VPWS, one is AToM, which target MPLS network and another is L2TPv3, which is a pseudo wire technology for resident IP network. Both AToM and L2TPv3 supports the transfer of ATM, HDLC, Frame-Relay and Ethernet traffic over an IP/MPLS network. PE routers run LDP protocol among them in an AToM implementation of Layer 2 technology. Pseudo wire or Tunnel is created between PE routers . This pseudo wire is used to transfer data between supplier edge routers. Two labels are associated with the data that travels from customer edge devices to provider edge device:

- Tunnel Label

- $\quad$ VC Label

The set of labels form the label stack. VC label is constantly the bottom label and Tunnel label is the top label in the label stack. The connection among PE router and the client edge router is called Attachment Circuit (AC). VC label identify to which addition circuit the frame or data belongs. VC label identifies the remote customer to to which data has been sent. The Tunnel label identifies the pseudo wire though data travels.

\section{Disadvantages:}

- Provide a diversified range of services (Layer 2, Layer 3 and Dial up VPNs) to meet the needs of the complete range of clients from tiny and average to Large business enterprise and economic institution.

- Make the service very simple for clients to use even if they lack familiarity in IP routing.

- Make the service very scalable and bendable to make possible large-scale deployment.

- Provide a consistent and acquiescent service.

- Offering SLA to clients.

- Capable of gathering a wide variety of client requirements, including security, quality of Service (QOS) and anyto-any connectivity and Capable of offering fully managed services to customer.

\section{PROPOSED SYSTEM}

\section{MPLS Layer 3 VPN:}

L3vpn provides IP and MPLS-based network virtualization solutions for enterprise and service provider customers. MPLS Layer 3 VPNs use a peer-to-peer model that uses Border Gateway Protocol (BGP) to dispense VPN-related information. This highly scalable, peer-to-peer model allows endeavor subscribers to outsource routing information to examine providers, resultant in considerable cost savings and a decrease in equipped complexity for enterprise. Service providers can then offer value-added services like Quality of Service (QoS) and Traffic Engineering ,Allowing network meeting that encompass voice, video, and data. IP-based VPNs use the next-generation Virtual Routing/Forwarding 
Vol. 8, Issue 3, March 2019

instance (VRF)-Lite, called effortless Virtual Network (EVN). This simplify Layer 3 system virtualization and allow clients to easily supply traffic separation and path separation on a shared network communications, remove the need to deploy MPLS in the endeavor network. EVN is fully incorporated with traditional MPLS-VPN or MPLS VPN MGRE.

\section{BGP - Border Gateway Protocol:}

\section{- $\quad$ RFC1771 (version 4)}

BGP is widely used across the Internet, but usually externally rather than internally. Interior use of BGP is classically only for very huge networks. For example, it might be used as a connection between multiple networks that are already using OSPF, when the whole network is too large for OSPF by itself. BGP is exclusive in that it uses TCP as its transport protocol. It is usually used as the protocol between Internet service provider. It include cost metrics for each path so that packets take the most efficient route. BGP is an exterior gateway protocol for communication between routers in unusual autonomous systems. BGP is base on a routing method called path-vector routing. Path-vector routing is different from both distance-vector routing and link-state outing. Path-vector routing does not have the volatility nor looping troubles of distance-vector routing. Each access in the routing table contain the destination network, the next router and the path to reach the destination. The pathway is regularly defined as an ordered list of independent systems that a packet should travel through to accomplish the destination. BGP is unusual from RIP and OSPF in that BGP uses TCP as its transport protocol. There are four types of BGP messages: open, update, keep alive and notification. BGP detect the collapse of either the connection or the host on the other end of the TCP connection by sending a keep alive message to its neighbor on a regular basis of the concept.

\section{Advantages:}

- $\quad$ MPLS -ATOM with virtual private LAN services can be used in large Enterprises by using MPLS network.

- $\quad$ High scalability - No limitation for number of nodes.

- $\quad$ No geographical limitation for the MPLS-ethernet network

\section{ETHERNET}

\section{ETHERNET TERMINOLOGY}

Ethernet follow a easy set of system that manage its essential operation. To better value these system, it is main to understand the fundamentals of Ethernet terminology.

Medium - Ethernet devices connect to a ordinary medium that provide a path along which the electronic signals will travel. Historically, this medium has been coaxial copper cable, but today it is more usually a twisted pair or fiber optic wiring.

Segment - We pass on to a single shared medium as an Ethernet segment.

Node - Devices that connect to that fragment are stations or nodes.

Frame - The nodes correspond in short communication called frames, which are variably sized chunk of information. The Ethernet protocol specify a set of system for construct frames. There are explicit smallest amount and limit lengths for frames, and a set of necessary pieces of information that must emerge in the frame. Each frame must contain, for example, both a objective address and a source address, which recognize the receiver and the sender of the message. The address exclusively identifies the node, just as a name identifies a particular person. No two Ethernet devices should ever have the same address.

\section{A Small Ethernet Network}

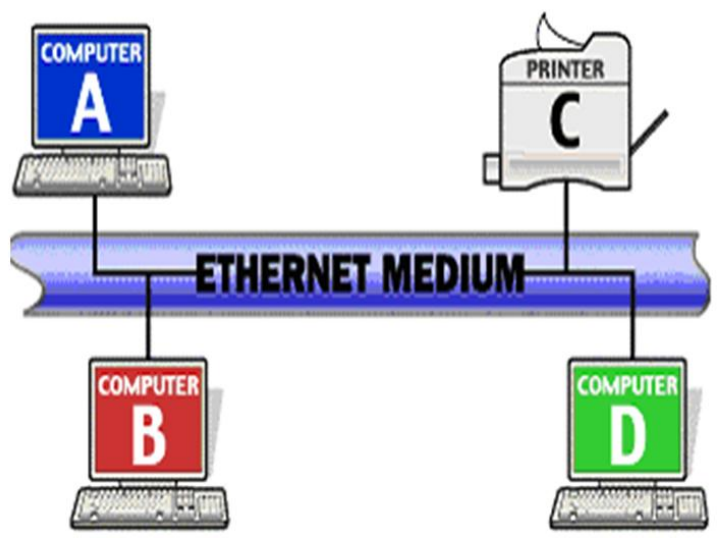


Vol. 8, Issue 3, March 2019

One exciting thing about Ethernet address is the implementation of a broadcast address. A frame with a destination address equivalent to the broadcast address (simply called a broadcast, for short) is planned for each node on the network, and each node will both collect and procedure this type of frame.

- Local Area Network (LAN) - A LAN is a network of computers that are in the same common physical position, typically within a structure or a property. If the computers are distant apart, then a Wide Area Network (WAN) is usually used.

- Network Interface Card (NIC) - all computer is associated to a network through an NIC. In mainly desktop computers, this is an Ethernet card (normally 10 or $100 \mathrm{Mbps}$ ) that is plug into a slot on the computer's motherboard.

- Media Access Control (MAC) address - This is the physical address of any device -- such as the NIC in a computer -- on the network. The MAC address has two parts, each 3 bytes long. The first 3 bytes classify the company that made the NIC. Unicast - A unicast is a transmission from one node addressed specifically to another node.

- Multicast - In a multicast, a node send a packet addressed to a particular collection address. Devices that are concerned in this group register to obtain packets addressed to the group. An example may be a CISCO router distribution out an inform to all of the other Cisco routers.

- Broadcast - In a broadcast, a node throw out a packet that is intentional for broadcast to all other nodes on the network.

\section{CONCLUSION}

In May 2014 Ezeh. G.N, Onyeakusi C.E, Adimonyemma T.M and Diala U.H. of Federal University of Technology approved out the proportional presentation assessment of Multimedia Traffic above Multiprotocol Label Switching using VPN and traditional IP networks. Association is finished on the basis(bits/seconds), end-to-enddelay(seconds)and utilization(tasks/sec).In this paper, outcome are analyze and it shows that MPLS provide better show in implement the VoIP application. In 2013 S.Venkata Raju, P.Premchand, A.Govardhan evaluate the Routing routine in Wide Area Networks using mpls, show best show of mpls in terms of throughput and end to end delay. MPLS is a label switching technology used mostly in Internet Service Provider(ISP) for label switching and VPN purpose. MPLS provide great concert with its label switching method. It also has the facility to create VPNs at both Layer 2 and Layer 3. In Layer 3 MPLS VPN, CE share the routing table information with the PE router, while in Layer 2 MPLS VPN, ISP act like a Layer 2 Switch and is used just to promote the packet from one CE to other. By default Layer 3 MPLS VPN has a junction time of 5-5.5 seconds, which can e concentrated to 2.5 to 3 seconds when we lock the SPF calculation of Link State IGP used in the core of ISP.

\section{REFERENCES}

[1]. Anderson, e.rosan “. Framework for layer 2 virtual private networks (12vpns)". Rfc 4664, september, 2006

[2]. 1. Martini, e. Rosen, t.smith" pseudowire setup and maintenance using the label distribution protocol (ldp)" april 2006

[3]. 1. Martini, g.heron "encapsulation methods for transport of ethernet over mpls networks." rfc4448, april (2006).

[4]. Armitage, grenville. "mpls: the magic behind the myths [multiprotocol label switching]." communications magazine, ieee 38.1 (2000): 124131.

[5]. Cisco," asr 9000 series 12 vpn and ethernet services configuration guide“, http://www.cisco.com/c/dam/en/us/td/I/300001400000/360001370000/361000362000/361074.eps/_jc r_content/renditions/361074.jpg

[6]. Sajassi, ali, et al. "bgp mpls based ethernet vpn." (2011).

[7]. Luo, wei, et al. layer 2 vpn architectures. pearson education, 2004.

[8]. Darukhanawalla, nash, et al. interconnecting data centers using vpls. cisco press, 2009.

[9]. Zhang, lixia, et al. "resource reservation protocol (rsvp)--version 1 functional specification." resource (1997). 